\title{
A Conformally Invariant Limit of the Critical Lattice Ising Model
}

\author{
Yu. A. Bashilov and S. V. Pokrovsky \\ Academy of Sciences, USSR, Space Research Institute, Profsoyuznaya 84/32, \\ SU-17810 Moscow, USSR
}

\begin{abstract}
A detailed analysis of an expansion in powers of $1 / N(N \gg 1)$ for the Hamiltonian and the transfer-matrix of the Ising chain consisting of $N$ sites is presented. A special attention is paid to a term proportional to $1 / N$ closely related to the theory of free massless Majorana fermions. An effective Hamiltonian isomorphic to that in conformally invariant theory is derived. The eigenvectors of the Ising Hamiltonian are classified in the framework of conformal algebra representation theory. The momentum and the energy for these states are expressed through the central charge and conformal dimensions. A similar relation for the logarithms of the transfer-matrix eigenvalues is ascertained. These are complex-valued functions of a spectral parameter $\theta$. A real part of such a function is shown to be proportional to $\sin \theta$, while an imaginary one - to $\cos \theta$. A direct geometrical interpretation of the lattice spectral parameter in the context of the conformally invariant theory for fermions inhabiting a torus is indicated. In other words, these fermions are represented as analytic anticommuting variables double (anti) periodic in a complex plane. The value of the spectral parameter coincides with an angle between these (anti) periods. A general scheme for the above expansion presumably suitable for a wide class of exactly solvable models is conjectured.
\end{abstract}

\section{Introduction}

A bootstrap program for classification of possible types of critical behavior in statistical systems proposed by Polyakov more than ten years ago [1,2] attracted new interest in the last few years. In the fundamental work by Belavin et al. [3], the conformal invariance in two dimensions (ultimately restrictive) was used together with bootstrap conditions to fix completely the operator algebra. Self-consistency conditions (locality plus associativity) combined with conformal invariance can be interpreted as a system of bootstrap equations. Any solution of this system represents some possible type of critical behavior. Though the problem of

\footnotetext{
$\star$ To whom all correspondence should be addressed
} 
classification is not exhausted, numerous bootstrap solutions are already found [3-7].

An important problem associated with conformal bootstrap is how to match lattice statistical models with a definite solution of conformal bootstrap equations. The simplest symmetry considerations were used to include the lattice Ising model and the 3-component Potts model into the bootstrap classification scheme $[3,4]$. The scaling exponents for the class of $Z_{n}$-symmetric lattice RSOS models were calculated analytically [8]. They were found to be identical with those found in the framework of parafermion conformal models [7].

A spectrum of dimensions together with a value of conformal anomaly (central charge) completely characterizes any conformally invariant theory. However, the analytical evaluation of scaling dimensions is rather difficult [9], especially when the exact solution exists only for the critical values of parameters [9-11].

Recently, Cardy et al. [12,13] related a transfer-matrix spectrum to the conformal characteristics and carried out a lot of numerical work. We develop their ideas in the context of the exactly solvable models intending to evaluate conformal invariants analytically. A sort of this calculation is given in the present paper.

Let the parameters of a lattice model fit their critical values. Then the logarithms of the transfer-matrix eigenvalues for a chain of a large but finite length $R$ can be expanded in powers of $1 / R(R \gg 1$ in lattice units) [12-14]

$$
\ln A_{n}=R f+f_{0}+\omega_{n} R^{-1}+O\left(R^{-2}\right) .
$$

The gaps separating the maximal eigenvalue from the nearest ones have the order of $1 / R$. The coefficient $f$ in (1.1) is simply the value of a bulk area density of free energy. The boundary term $f_{0}$ vanishes for the cyclic boundary conditions. The amplitudes of a finite size scaling $\omega_{n}$ are universal [12-14]. Every transfer-matrix eigenvector $|n\rangle$ (with the eigenvalue close to the maximal one) corresponds to some state in the conformal algebra representation. Denote the central charge for the whole representation by " $c$ " and by $\Delta_{n}, \bar{\Delta}_{n}$ - the analytic and antianalytic dimensions of the particular conformal state $|n\rangle$. Then $\omega_{n}$ can be expressed through these quantities in the following way:

$$
w_{n}=-2 \pi v\left[\cos \alpha\left(\Delta_{n}+\bar{\Delta}_{n}-\frac{c}{12}\right)+i \sin \alpha\left(\Delta_{n}-\bar{\Delta}_{n}\right)\right] .
$$

Here the parameters $\alpha$ and $v$ depend on the lattice model characteristics (see Sect. 2). In particular, the phase $\alpha$ depends on the value of the transfer-matrix spectral parameter $\theta$. We have found for the Ising model $\alpha=\pi / 2-\theta$, and we argue that this is valid in general. Note that $\omega_{n}$ takes complex values because the transfermatrix is non-Hermitian at the arbitrary values of its spectral parameter $\theta$.

Let us give an outline of the following sections. In Sect. 2. we discuss some general features of a transier-matrix spectrum and its relation (1.2) to the conformal invariants. In the next sections these considerations plausible for any exactly solvable model undergo detailed verification for the case of the Ising model. In Sect. 3 a conformally invariant counterpart of the lattice Ising model, i.e. a theory of free massless Majorana fermions, is described. In Sect. 4, to clarify the 
connection between these two models, we review a diagonalization of the Ising chain Hamiltonian. An effective lattice Hamiltonian derived for the low lying excitations coincides with the regularized conformally invariant one. However, the boundary conditions for the spin chain rule out half of the fermionic states. In Sect. 5 we examine the expansion (1.1) for the eigenvalues of the transfer-matrix commuting with the Hamiltonian. The formula (1.2) is confirmed and the connection between $\alpha$ and $\theta$ is ascertained. In Sect. 6 we study a theory of free massless Majorana fermions with periodic or antiperiodic boundary conditions in the arbitrary spaced parallelogram. The conformally invariant free energy depends only on the ratio of the lengths of the periods and the angle between them. The latter is shown to be equal to the spectral parameter $\theta$ of the lattice model. When one of the periods tends to infinity, the strip geometry results described by (1.2) are reproduced. Namely, the value of free energy per unit length of an infinitely long strip is given by (1.2) when: 1) $\Delta_{n}=\bar{\Delta}_{n}=0$ and the fermions are antiperiodic across the strip; 2) $\Delta_{n}=\bar{\Delta}_{n}=1 / 16$ and the fermions are periodic. In Sect. 7 we briefly discuss which of the results for the Ising model can be generalized for a wider class of the exactly solvable models.

\section{The Conformally Invariant Limit for the Exactly Solvable Lattice Statistical Models}

It is well known that the lattice model belongs to the class of exactly solvable ones if its transfer-matrix (TM) commutes with the whole parametric family of TM. This family can be regarded as the matrix-valued function $\mathbf{V}(\theta)$ of the spectral parameter $0 \leqq \theta \leqq \pi[9,10]$. The quantum Hamiltonian for such a system is defined through the logarithmic derivative of $\operatorname{TMV}(\theta)$ at $\theta=0[9,10]$,

$$
\text { 甚 }=\left.\mathbf{V}^{-1}(\theta) \frac{d}{d \theta} \mathbf{V}(\theta)\right|_{\theta=0} \text {. }
$$

The Hamiltonian (2.1) commutes with the whole family $\mathbb{V}(\theta)$ and usially is Hermitian. The total family of TM V $(\theta)$ may be diagonalized in the basis formed by the eigenvectors of the Hamiltonian (2.1). Note that this basis is independent on the spectral parameter. The Hamiltonian eigenvalues $E_{n}$ may be expressed through the logarithmic derivatives of TM eigenvalues (TME) $\Lambda(\theta)$ at $\theta=0$

$$
E_{n}=-\left.\frac{d}{d \theta} \ln \Lambda_{n}(\theta)\right|_{0=0}
$$

Any member of the family and the Hamilionian commute with the lattice translation operator exp $(-i \mathbf{P})$. The last can be diagonalized by an appropriate rearrangement of the basis described above. The operator in the exponent is the momentum operator. It is adopted that low Jying excited states of the Hamiltonian are combinations of a finite number of elementary excitations. The momentum or the energy of a given state $|n\rangle$ breaks into the sum of elementary momenta or energies

$$
p_{n}=\sum_{l} p_{j} ; \quad E_{n}-E_{0}=\sum_{i} c\left(p_{j}\right)
$$


The functions $\varepsilon(p)$ for different types of excitations do not coincide in general. The wave function of an elementary excitation is proportional to the exponent

$$
\exp \left\{-\varepsilon\left(p_{j}\right) t-i p_{j} x\right\}
$$

where $x$ denotes the coordinate along the chain and $t$ denotes Euclidean time. These exponents can be considered as conformally invariant when $\varepsilon(p)$ is gapless,

$$
\varepsilon(p)=v|p|
$$

and the velocity $v$ is the same for all types of excitations. Note that only the longwave excitations $(p \simeq 1 / R)$ should be considered. Then only the first term in the expansion of $\varepsilon(p)$ in powers of $p$ should be left. The positivity of an excitation energy is accounted for by writing the absolute value of $p$. All excitations with a linear dispersion law (2.4) break into two groups. The right (analytic) have $p>0$ and depend on a single complex variable $z=v t+i x$. The left (antianalytic) have $p<0$ and depend on the complex conjugate variable $\bar{z}=v t-i x$. The momentum and energy of a given state $|n\rangle$ can be represented in the following form:

$$
P_{n}=\sum_{r} p_{r}-\sum_{l} q_{l} ; \quad E_{n}-E_{0}=v \sum_{r} p_{r}+v \sum_{l} q_{l} .
$$

Here $p_{r}$ and $q_{l}$ denote the absolute values of the momenta for the right and the left excitations, respectively. The expressions (2.5) should be compared with that in the conformally invariant theories [3]. We shall describe briefly some basic points in the Hamiltonian formalism for these theories. The general coordinate transformations are generated by a symmetric stress tensor (ST) $T_{\mu \nu}(\mathbf{x})$. Coordinate covariance of the theory is guaranteed by the conservation law: $\partial_{\mu} T_{\mu \nu}(\mathbf{x})=0$. The system is scale invariant if the $\mathrm{ST}$ is traceless: $T_{\mu \mu}(\mathbf{x})=0$. But since this requirement is imposed, a more general conformal invariance arises. In two dimensions, symmetric and traceless, ST has only two independent components that may be expressed through the analytic function $T(z)$ and the antianalytic one $\bar{T}(\bar{z})$ due to the conservation law [3],

$$
\begin{gathered}
T_{\tau \tau}(\tau, \sigma)=-T_{\sigma \sigma}(\tau, \sigma)=T(z)+\bar{T}(\bar{z}) \\
T_{\sigma \tau}(\tau, \sigma)=i T(z)-i \bar{T}(\bar{z}) .
\end{gathered}
$$

Here $z$ and $\bar{z}$ are complex coordinates connected with the Cartesian ones $(\tau, \sigma)$ in a usual manner

$$
z=\tau+i \sigma ; \quad \bar{z}=\tau-i \sigma .
$$

One can expand ST into the formal Fourier series in the interval $0 \leqq \sigma \leqq R$ with periodic boundary conditions. Due to its analyticity

$$
T(z)=\left(\frac{2 \pi}{R}\right)^{2} \sum_{n=-\infty}^{\infty} \mathbf{L}_{n} \exp \left(-\frac{2 \pi}{R} n z\right) .
$$

The same expansion is valid for antianalytic component $\bar{T}(\bar{z})$ after $z$ is substituted by $\bar{z}$ and $\mathbf{L}_{n}$ by $\overline{\mathbf{L}}_{n}$. The Fourier coefficients $\mathbf{L}_{n}$ in (2.9) form a Virasoro algebra with a central charge " $c$ " [3]. The direct sum of two Virasoro algebras (that of $\mathbf{L}_{n}$ and another of $\mathbf{L}_{n}$ ) is the algebra of conformal transformations [3]. The arbitrary infinitesimal conformal transformation of coordinates, resulting in 
analytic variation of $z$ by $\alpha(z)$ and antianalytic variation of $\bar{z}$ by $\bar{\alpha}(\bar{z})$, is generated by the operator $\mathbf{T}_{\alpha}+\overline{\mathbf{T}}_{\bar{\alpha}}$, where

$$
\mathbf{T}_{\alpha}=\int_{0}^{R} \frac{d \sigma}{2 \pi} \alpha(z) T(z) ; \quad \overline{\mathbf{T}}_{\bar{\alpha}}=\int_{0}^{R} \frac{d \sigma}{2 \pi} \bar{x}(\bar{z}) \bar{T}(\bar{z}) .
$$

By virtue of (2.10) one can express the operator of momentum and the Hamiltonian generating translations along the coordinates $\sigma$ and $\tau$ respectively through the conformal generators $L_{0}$ and $\bar{L}_{0}$,

$$
\mathbf{P}=\frac{2 \pi}{R}\left(\mathbf{L}_{0}-\mathbf{L}_{0}\right) ; \quad \mathbf{H}=\frac{2 \pi}{R}\left(\mathbf{L}_{0}+\mathbf{L}_{0}\right) .
$$

The Virasoro algebra representation called Verma modulus grows from the highest weight vector (ancestor) [3]. The ancestor annihilated by Virasoro generators $\mathbf{L}_{n}$ with positive $n$ is an eigenvector of $\mathbf{L}_{0}$ :

$$
\begin{aligned}
& \mathbf{L}_{n}|\Delta\rangle=0, \quad n>0 ; \\
& \mathbf{L}_{0}|\Delta\rangle=\left(\Delta-\frac{c}{24}\right)|\Delta\rangle .
\end{aligned}
$$

Here $\Delta$ is a conformal dimension (weight) of the representation consisting of the ancestor $|\Delta\rangle$ and its descendants created by a product of some $\mathbf{L}_{n}$ with negative $n$ acting on $|\Delta\rangle$. Any given descendant is also annihilated by all $\mathbf{L}_{n}$ with $n$ exceeding some positive integer and has a definite conformal dimension equal to the sum of $\Delta$ and another positive integer.

We draw the reader's attention to the difference in notations for the ancestors in (2.12) and that by Belavin et al. [3], where instead of our $(\Delta-c / 24)$ simply $\Delta$ appears. This difference has a geometrical origin. Our $T(z)$ acts on the strip of width $R$ with periodic boundary conditions while in [3] the ST $\widetilde{T}(\zeta)$ acts on the whole complex plane of $\zeta$. One can transform a strip to a plane by an exponential map

$$
\zeta=\exp \left(\frac{2 \pi}{R} z\right)
$$

A finite analytic coordinate transformation $\zeta(z)$ causes the following transformation of the ST [3],

$$
T(z)=\left(\frac{d \zeta}{d z}\right)^{2} \widetilde{T}(\zeta(z))+\frac{c}{12}\{\zeta, z\},
$$

where $\{\zeta, z\}$ stands for the Schwartzian

$$
\{\zeta, z\}=\left(\frac{d^{3} \zeta}{d z^{3}}\right) /\left(\frac{d \zeta}{d z}\right)-\frac{3}{2}\left(\frac{d^{2} \zeta}{d z^{2}}\right)^{2} /\left(\frac{d \zeta}{d z}\right)^{2} .
$$

Virasoro generators in [3] are defined as the coefficients in the Laurent expansion of $\widetilde{T}(\zeta)$

$$
\widetilde{T}(\zeta)=\sum_{n=-\infty}^{\infty} \widetilde{L}_{n} \zeta^{-(n+2)}
$$


One can express $\mathbf{L}_{n}$ through $\tilde{\mathbf{L}}_{n}$ substituting (2.13) into (2.14) and accounting for (2.16).

$$
\mathbf{L}_{n}=\tilde{\mathbf{L}}_{n}-\mathbf{I} \frac{c}{24} \delta_{n, 0}
$$

An antianalytic component of the ST $\bar{T}(\bar{z})$ can be treated in the same manner. Returning to the momentum and the Hamiltonian in the conformally invariant theory we find due to (2.11) and (2.12) that in Verma modulus an ancestor and any of its descendants $|n\rangle$ having definite conformal dimensions $\Delta_{n}$ and $\bar{\Delta}_{n}$ is the eigenvector of both operators. The momentum and the energy may be expressed through the conformal dimensions in the following way:

$$
P_{n}=\frac{2 \pi}{R}\left(\Delta_{n}-\bar{\Delta}_{n}\right) ; \quad E_{n}=\frac{2 \pi}{R}\left(\Delta_{n}+\bar{\Delta}_{n}-\frac{c}{12}\right) .
$$

The formulas (2.18) agree with (2.5) after renormalization of the energy in (2.5). $E_{n} \rightarrow v^{-1} E_{n}(\tau=v t)$ and give the expressions for the dimensions

$$
\Delta_{n}=\frac{R}{2 \pi} \sum_{r} p_{r} ; \quad \bar{\Lambda}_{n}=\frac{R}{2 \pi} \sum_{l} q_{l} .
$$

The lattice value of the ground state energy may be divided into the universal part proportional to $1 / R$ and the nonuniversal one proporitional to $R$

$$
E_{0}=R \varepsilon-\frac{\pi c}{6 R} v+O\left(R^{-2}\right)
$$

While long-wave degrees of freedom contribute to the universal part, the shortwave ones contribute to the thermodynamic value of energy density $\varepsilon$. Higher order terms represent the corrections to scaling.

The connection between the values of energy and momentum for the excitations and the conformal dimensions was revealed by Cardy [12]. The formula similar to (2.20) appeared in [13].

Let us derive the corresponding formulas for TME. At the arbitrary value of its spectral parameter the TM is non-Hermitian. Its eigenvalues are complex, hence the amplitudes $\omega_{n}$ in (1.2) are also complex.

When the evolution of a system is governed by the TM instead of the Hamiltonian, a role of the excitation energy $E_{n}-E_{0}$ is played by the quantity $\Omega_{n}=-\ln \left(\Lambda_{n} / \Lambda_{0}\right)$. A large time asymptotic for the eigenvector $|n\rangle$ is proportional to $\exp \left(-\Omega_{n} t\right)$. One might expect the expansions for $\Omega_{n}$ like (2.5) to exist for the low lying excited states. However, the "velocities" in this case may be complex,

$$
\Omega_{n} \equiv \ln \left(\Lambda_{0} / \Lambda_{n}\right)=v e^{i \alpha} \sum_{r} p_{r}+v e^{-i \alpha} \sum_{l} q_{l} .
$$

The range of the variation for a parameter $\alpha$ is chosen to guarantee the positivity of $\operatorname{Re} \Omega_{n}$. Now we shall elucidate how the amplitudes (2.21) arise in the framework of the conformally invariant field theory. An elementary right excitation with a definite value of momentum $p$ behaves as $\exp (-p z)$, where

$$
z \equiv \tau+i \sigma=v e^{i \alpha} t+i x .
$$




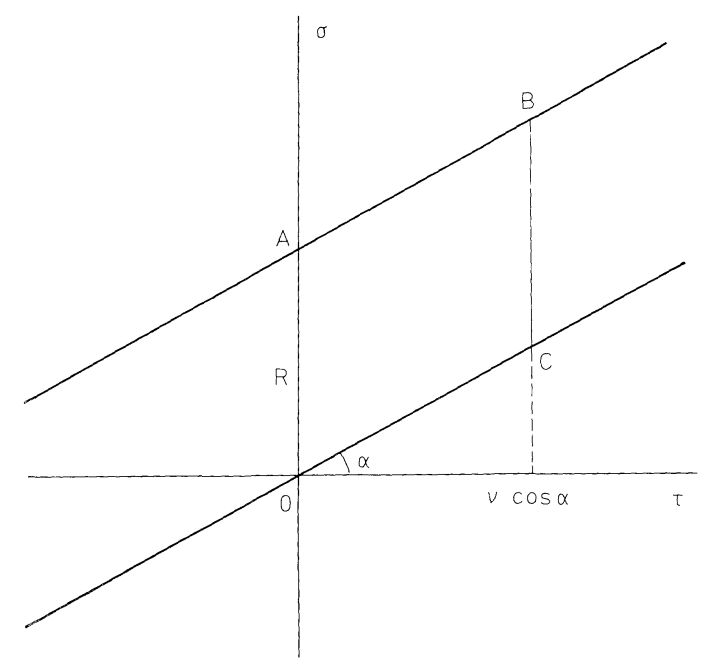

Fig. 1. A strip in a Cartesian frame $(\tau, \sigma)$ of Euclidean space is an image of a strip of width $R$, which is horizontal in the initial coordinates $(x, t)$. The coordinate transformation (2.23) maps a rectangular of a unit length cutout of the initial strip onto the parallelogram $\mathrm{OABC}$. The lengths of the sides $\mathrm{OA}$ and $\mathrm{OC}$ are equal to $R$ and $v$, respectively

The left one with a momentum " $-q$ " behaves as $\exp (-q \bar{z})$, where $\bar{z}$ is a complex conjugate to $z$. The coordinates $\tau$ and $\sigma$ are Cartesian

$$
\tau=v \cos \alpha t ; \quad \sigma=v \sin \alpha t+x .
$$

The initial strip horizontal in $(x, t)$-frame inclines in the Cartesian coordinates $\tau, \sigma$ making an angle $\alpha$ with the axis of abscissas (Fig. 1). The rectangle $0 \leqq t<1$; $0 \leqq x<R$ maps under transformation (2.23) into the parallelogram $\mathrm{OABC}$ with the area equal to $R v \cos \alpha$.

The TME logarithm $\ln A_{n}$ can be treated as a free energy per unit length for the system on the strip with some complex boundary conditions imposed at $t \rightarrow \pm \infty$. To find the dependence of this quantity on a width $R$ of the strip, one should perform an infinitesimal coordinate transformation changing $R$ by $\delta R$ and leaving the boundary conditions intact. The response of the system to the coordinate variation $\alpha_{\mu}(\mathbf{x})$ results in the variation of a free energy by the quantity

$$
\delta \ln \Lambda_{n}=-\int\left\langle n\left|T_{\mu v}(\mathbf{x})\right| n\right\rangle \hat{\partial}_{\mu} \alpha_{v} \frac{d^{2} x}{2 \pi} .
$$

We emphasize that this formula is valid for the Euclidean space in a Cartesian frame.

A transformation leading to variation of width by $\delta R$ is simply a rescaling of $x$ by the factor $(1+\delta R / R)$. This dilatation induces a linear transformation of the Cartesian coordinates in accordance with (2.23)

$$
\delta \tau=0 ; \quad \delta \sigma=(\delta R / R)(\sigma-\tau \tan \alpha) .
$$


The response of the free energy per unit length of the strip is due to $(2.24)$

$$
\delta \ln \Lambda_{n}=-\frac{1}{2 \pi}\left(\frac{\delta R}{R}\right) \delta\left\langle n\left|T_{\sigma \sigma}-T_{\tau \sigma} \tan \alpha\right| n\right\rangle d \sigma d \tau,
$$

where the integration is extended over the area of the parallelogram $\mathrm{OABC}$ (Fig. 1). The periodic boundary conditions across the strip imposed on the system in the initial lattice coordinates should be traced in the periodicity along the coordinate $\sigma$ with a period $R$. The integration over $\sigma$ leaves only zero Fourier harmonics of the ST in (2.26),

$$
\delta \ln \Lambda_{n}=2 \pi v\left(\frac{\delta R}{R^{2}}\right)\left[\cos \alpha\left\langle n\left|L_{0}+\bar{L}_{0}\right| n\right\rangle+i \sin \alpha\left\langle n\left|L_{0}-\bar{L}_{0}\right| n\right\rangle\right] .
$$

Removing the variation and substituting (2.12) one readily gets (1.2).

\section{The Conformally Invariant Theory of Free Fermions}

The model of free Majorana fermions is the conformally invariant counterpart of the lattice Ising model $[3,4,7]$. The basic objects in it are the two conserving fermion currents $\psi(z)$ and $\bar{\psi}(\bar{z})$. Here $z$ and $\bar{z}$ are complex coordinates on a strip $0 \leqq \sigma<R ;-\infty<\tau<\infty$. The coordinates $(\tau, \sigma)$ are Cartesian connected with $z$ and $\bar{z}$ by (2.9). The Fock space of all fermionic states consists of two sectors. The NeveuSchwartz (NS) sector contains the Fock space of the antiperiodic fermions while in the Ramond sector the fermions are periodic [15]

$$
\begin{aligned}
& \psi(\tau, \sigma+R)= \pm \psi(\tau, \sigma) ; \\
& \bar{\psi}(\tau, \sigma+R)= \pm \bar{\psi}(\tau, \sigma) .
\end{aligned}
$$

The equal time commutation relations must agree with the (anti) periodicity conditions

$$
\begin{gathered}
\left\{\psi(\tau, \sigma), \psi\left(\tau, \sigma^{\prime}\right)\right\}=\left\{\bar{\psi}(\tau, \sigma), \bar{\psi}\left(\tau, \sigma^{\prime}\right)\right\}=2 \pi \delta^{( \pm)}\left(\sigma-\sigma^{\prime}\right) \\
\left\{\psi(\tau, \sigma), \bar{\psi}\left(\tau, \sigma^{\prime}\right)\right\}=0 .
\end{gathered}
$$

Here $\delta^{( \pm)}(\sigma)$ denotes the (anti) periodic Dirac $\delta$-function with a period $R$. The (anti) periodic fermions possess the following Fourier expansion:

$$
\psi(z)=\left(\frac{2 \pi}{R}\right)^{1 / 2} \sum_{v} \psi_{v} \exp \left(-\frac{2 \pi}{R} v z\right),
$$

where the sum is extended over all half-odd-integers in the NS sector and over all integers in the Ramond sector. The same Fourier expansion can be written for $\bar{\psi}(\bar{z})$ with a substitution $z$ by $\bar{z}$ and $\psi_{v}$ by $\bar{\psi}_{v}$. The Fourier harmonics by virtue of (3.2) and (3.3) obey the following commutation relations

$$
\left\{\psi_{\mu}, \psi_{v}\right\}=\left\{\bar{\psi}_{\mu}, \bar{\psi}_{v}\right\}=\delta_{\mu+v .0} ; \quad\left\{\psi_{\mu}, \bar{\psi}_{v}\right\}=0 .
$$

In particular, $\psi_{0}^{2}=1 / 2$ in the Ramond sector. In the Euclidean space-time manifold fermions undergo conjugation if time is reversed: $\boldsymbol{\psi}(-\tau, \sigma)=\boldsymbol{\psi}^{+}(\tau, \sigma)$. That results 
in the following relations between their Fourier harmonics:

$$
\boldsymbol{\psi}_{v}^{+}=\boldsymbol{\psi}_{-v} ; \quad \overline{\boldsymbol{\psi}}_{v}^{+}=\overline{\boldsymbol{\psi}}_{-v} .
$$

A representation of the fermion current algebra in NS sector grows out of a single referring state $|0\rangle$ called vacuum,

$$
\psi_{v}|0\rangle=0, \quad v>0 .
$$

The descendants of the vacuum are created by the action on of a finite number of $\psi_{v}^{+}(v>0)$,

$$
\left|v_{1}, v_{2}, \ldots, v_{N}\right\rangle=\boldsymbol{\psi}_{v_{N}}^{+}, \ldots, \boldsymbol{\psi}_{v_{2}}^{+} \boldsymbol{\psi}_{v_{1}}^{+}|\mathbf{0}\rangle ; \quad v_{1}>v_{2}>v_{3}>\ldots>v_{N} .
$$

The vacuum vector in the Ramond sector is two-fold degenerate because $\boldsymbol{\psi}_{0}^{2}=1 / 2$ exists. We denote two vacuum vectors by $|\sigma\rangle$ and $|\mu\rangle$. They obey the following requirements:

$$
\begin{gathered}
\boldsymbol{\psi}_{v}|\sigma\rangle=\boldsymbol{\psi}_{v}|\mu\rangle=0, \quad v>0 \\
\boldsymbol{\psi}_{0}|\sigma\rangle=2^{-1 / 2} e^{i \alpha}|\mu\rangle ; \quad \boldsymbol{\psi}_{0}|\mu\rangle=2^{-1 / 2} e^{-\mid \alpha}|\sigma\rangle ;
\end{gathered}
$$

The descendants of $|\sigma\rangle$ and $|\mu\rangle$ are created by Ramond operators $\boldsymbol{\psi}_{v}^{+}(v>0)$ acting on the vacuum vectors in the same manner as in (3.7).

To simplify the notations, only the analytic fermion $\psi(z)$ representation was described. The indices responsible for the antianalytic structure were suppressed. However, the reconstruction of the antianalytic structure is rather obvious.

An operator product expansion for the analytic fermions has the following form $[4,7]$

$$
\psi(z+\varepsilon / 2) \psi(z-\varepsilon / 2)=\varepsilon^{-1}+2 \varepsilon T(z)+O\left(\varepsilon^{3}\right),
$$

where $T(z)$ is an analytic part of ST. The same expansion can be written for antianalytic $\bar{\psi}(\bar{z})$.

Let us compare (3.10) with the expression for the operator product, where a singular part is picked out by a normal ordering,

$$
\psi(z+\varepsilon / 2) \psi(z-\varepsilon / 2)=\frac{\pi}{R}\left\{\begin{array}{c}
\operatorname{cosech}\left(\frac{\pi \varepsilon}{R}\right) \\
\operatorname{coth}\left(\frac{\pi \varepsilon}{R}\right)
\end{array}\right\}+: \psi(z+\varepsilon / 2) \psi(z-\varepsilon / 2): .
$$

The higher expression in the braces corresponds to the NS sector while the lower to the Ramond sector. Normal ordering standardly denoted by double vertical dots means such an arrangement of $\boldsymbol{\psi}_{v}$ in the product that the indices $v$ increase from the left to the right. Normal ordered terms in (3.12) are regular in $\varepsilon$,

$$
\begin{gathered}
: \psi(z+\varepsilon / 2) \psi(z-\varepsilon / 2): \\
=\frac{4 \pi}{R} \sum_{v_{1}<v_{2}} \boldsymbol{\psi}_{v_{1}} \boldsymbol{\psi}_{v_{2}} \sinh \left[\frac{\pi \varepsilon}{R}\left(v_{2}-v_{1}\right)\right] \exp \left[-\frac{2 \pi}{R} z\left(v_{1}+v_{2}\right)\right] .
\end{gathered}
$$


Recall that summation is different in NS and Ramond sectors (see the prescription after (3.3)). Extracting the linear in 8 term out of the right-hand side of (3.11) one gets the expression for $T(z)$. We present here the explicit formulas for Fourier harmonics $L_{n}$ that are very similar in NS and Ramond sectors,

$$
\mathbb{Z}_{n}=\frac{1}{2} \sum_{v_{1}+v_{2}=n}\left(v_{2}-v_{1}\right): \psi_{v_{1}} \psi_{v_{2}}: \quad n \neq 0,
$$

and

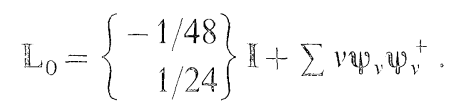

The higher number in braces applies to the NS sector while the lower one applies to the Ramond sector. The summation is also different in NS and Ramond sectors (see the prescription just after (3.3)).

Antianalytic Virasoro operators $\mathbb{L}_{n}$ possess the same representation (3.13), (3.14), where $\psi_{v}$ should be substituted by $\bar{\psi}_{v}$.

The fermionic Fock space decomposes under action of conformal algebra into the direct sum of several irreducible representations.

Let us consider first an action of the analytic Virasoro algebra on the NS sector. We suppress antianalytic indices of vectors as previously.

Any given Virasoro generator $\mathrm{L}_{n}$ is bilinear in fermion operators $\boldsymbol{\psi}_{v}$ (see (3.13), (3.14)) and consequently has non-vanishing elements between two states (3.7) with an equal number of fermions, all indices $v_{j}$ except one coinciding. The residual between the deviating indices is equal to $n$. Another type of the nonvanishing matrix elements is that between the vector (3.7) and another one, where the number of fermions is increased or decreased by two. The sum of the quantum numbers $v$ for these extra fermions must be equal to $n$. Thus a linear span of the vectors (3.7) with even numbers of fermions constitute a representation of Virasoro algebra. Its highest weight vector is a fermionic vacuum $|0\rangle$ conciding with a true conformal vacuum [3] $\left(\mathbf{L}_{0}|0\rangle=\mathbb{L}_{-1}|0\rangle=0\right)$. Another Verma modulus is constructed of the vectors (3.7) with an odd number of fermions. Its ancestor is a vector $|\psi\rangle \equiv|1 / 2\rangle$ having the dimension $\Delta=1 / 2$. Restoring the antianalytic structure, one sees that the fermionic Fock space splits into the following four irreducible representations of the complete conformal algebra:

$\begin{array}{lllll}\text { ancestor } & |0\rangle & |\psi\rangle & |\bar{\psi}\rangle & |\varepsilon\rangle \\ & |0, \overline{0}\rangle & |1 / 2, \overline{0}\rangle & |0, \overline{1} / 2\rangle & |1 / 2, \overline{1} / 2\rangle \\ \text { number of analytic fermions } & \text { even } & \text { odd } & \text { even } & \text { odd } \\ \text { number of antianalytic fermions } & \text { even } & \text { even } & \text { odd } & \text { odd. }\end{array}$

Here indices without bars denote the analytic quantum fermionic numbers while indices with bars specify the antianalytic ones.

Let us proceed to the Ramond sector of the fermionic Fock space. Acting on the highest weight vector $|\sigma\rangle$ by Virasoro generators one can obtain any vector $\left|\sigma ; v_{1}, \ldots, v_{2 N}\right\rangle$ with an even number of fermions. But since $v_{j}$ are decreasing nonnegative integers (in analogy with (3.7)), then $v_{2 N}$ may turn into zero. This state belongs to the array of the vectors $\left|\mu ; v_{1}, \ldots, v_{2 N-1}\right\rangle$ with an odd number of fermions. Interchanging formally $\sigma$ and $\mu$ one gets another Verma modulus. 
The complete conformal algebra decompose the Ramond sector into two irreducible representations. The first has the ancestor $|\sigma\rangle$ and contains the vectors of two kinds. The vectors of the form $|\sigma:\{v\} ;\{\bar{v}\}\rangle$ with the numbers of analytic and antianalytic fermion of the same evenness constitute the first multitude. The second one contains the vectors of the form $\langle\mu ;\{v\} ;\{\bar{v}\}\rangle$ with the numbers of analytic and antianalytic fermions of a different evenness. In both cases the indices $v$ and $\bar{v}$ (analytic and antianalytic respectively) must be positive integers.

The interchange of $\sigma$ and $\mu$ in the construction described above gives the second irreducible representation.

We conclude this section with the formulas for the momentum and energy of the state with a set of fermionic quantum numbers $\{v\}$ and $\{\vec{v}\}$ analytic and antianalytic respectively.

$$
\begin{gathered}
P_{\{v \cdot \bar{v}\}}=\frac{2 \pi}{R} \sum_{j=1}^{N} v_{j}-\frac{2 \pi}{R} \sum_{j=1}^{N} \bar{v}_{j} ; \\
E_{\{v, \bar{v}\}}=\left\{\begin{array}{c}
-\pi / 12 R \\
\pi / 6 R
\end{array}\right\}+\frac{2 \pi}{R} \sum_{j=1}^{N} v_{j}+\frac{2 \pi}{R} \sum_{j=1}^{N} \bar{v}_{j} .
\end{gathered}
$$

These formulas are suitable for both NS and Ramond sectors provided that the non-negative quantum numbers are taken as half-odd-integers and integers respectively. The higher number in the braces of (3.16) corresponds to the NS sector while the lower one corresponds to the Ramond sector.

\section{The Rettice Hamiltonian and Fermions}

Consider an Ising Hamiltonian on the one-dimensional chain consisting of $2 \mathrm{~N}$ sites,

$$
\mathbb{H}=\frac{1}{2} \sum_{n=1}^{2 N}\left(\sigma_{n+1}^{z} \sigma_{n}^{z}+k \sigma_{n}^{x}\right) ; \quad \sigma_{2 N+1}^{z}=\sigma_{1}^{z}
$$

where the operators $\mathbb{\sigma}_{n}^{x}, \mathbb{F}_{n}^{z}$ located on different sites commute and generate a standard algebra of Pauli matrices when located on the same site. The Hamiltonian (4.1) commutes with an operator of reversal of all spins

$$
\mathbb{R}=\prod_{n=1}^{2 N} \sigma_{k}^{x} ; \quad \mathbb{R}^{2}=\mathbb{I}
$$

The Hamiltonian (4.1) may be diagonalized in three steps [16].

Jordan-Wigner Transformation. The Majorana fermions instead of spin operators are introduced in the following way:

$$
\varphi_{n}=\sigma_{n}^{z} \prod_{k=1}^{n-1} \sigma_{k}^{x} ; \quad \chi_{n}=\sigma_{n}^{y} \prod_{k=1}^{n-1} \sigma_{k}^{x} .
$$

These fermions form the Clifford algebra

$$
\left\{\Phi_{n}, \oplus_{m}\right\}=\left\{\chi_{n}, \chi_{m}\right\}=2 \delta_{n, m} ; \quad\left\{\Phi_{n}, \chi_{m}\right\}=0
$$


The Hamiltonian (4.1) is bilinear in the fermion variables.

$$
\begin{aligned}
\mathbf{H} & =\mathbf{H}_{+} \frac{\mathbf{I}+\mathbf{R}}{2}+\mathbf{H}_{-} \frac{\mathbf{I}-\mathbf{R}}{2} ; \\
\mathbf{H}_{ \pm} & =\frac{i}{2} \sum_{n=1}^{2 N}\left(\boldsymbol{\varphi}_{n+1}-k \boldsymbol{\varphi}_{n}\right) \boldsymbol{\chi}_{n} .
\end{aligned}
$$

Here the lower indices " \pm " mean the fermions to be continued out of the interval $1<n<2 N$ antiperiodically or periodically respectively. Note that the commutation relations (4.4) should respect the periodicity conditions in each sector $\mathrm{R}= \pm 1$. The Heisenberg equations of motion governed by the Hamiltonian (4.1) for the fermions $\varphi_{n}$ and $\chi_{n}$ are linear and translationally invariant [17]. That is why they are much simplified in the momentum representation.

The Momentum Fourier Representation.

$$
\boldsymbol{\varphi}_{n}=N^{-1 / 2} \sum_{v} \tilde{\varphi}_{v} e^{-i n p_{v}} ; \quad \chi_{n}=N^{-1 / 2} \sum_{v} \tilde{\chi}_{v} e^{-i n p_{v}},
$$

where due to the periodicity conditions

$$
p_{v}=\frac{\pi}{N} v:\left\{\begin{array}{lll}
v=-N+\frac{1}{2},-N+\frac{3}{2}, \ldots, N-\frac{1}{2} & \text { if } \quad \mathrm{R}=1 ; \\
v=-N+1,-N+2, \ldots, N-1, N & \text { if } \quad \mathrm{R}=-1 .
\end{array}\right.
$$

The Fourier harmonics $\tilde{\varphi}_{v}, \tilde{\chi}_{v}$ commute identically in both sectors $R= \pm 1$,

$$
\left\{\tilde{\boldsymbol{\varphi}}_{v}, \tilde{\boldsymbol{\varphi}}_{\mu}\right\}=\left\{\tilde{\boldsymbol{\chi}}_{v}, \tilde{\boldsymbol{\chi}}_{\mu}\right\}=\dot{\delta}_{\mu+v, 0} ; \quad\left\{\tilde{\boldsymbol{\varphi}}_{v}, \tilde{\boldsymbol{\chi}}_{\mu}\right\}=0,
$$

where $v$ and $\mu$ take integer values $(R=-1)$ and half-odd-integer ones $(R=+1)$ simultaneously and

$$
\delta_{\mu+v, 0}=1 \text { iff } \mu+v=0(\bmod 2 N) .
$$

As the Majorana fermions $\tilde{\varphi}_{n}$ and $\tilde{\chi}_{n}$ in coordinate representations are Hermitian it turns out that their Fourier harmonics $\varphi_{v}, \chi_{v}$ obey certain conjugation relations

$$
\tilde{\boldsymbol{\varphi}}_{v}^{+}=\tilde{\boldsymbol{\varphi}}_{-v} ; \quad \tilde{\chi}_{v}^{+}=\tilde{\chi}_{-v} .
$$

In particular, four self-conjugate operators exist in the $\mathrm{R}=-1$ sector: $\tilde{\boldsymbol{\varphi}}_{0}, \tilde{\chi}_{0}, \tilde{\boldsymbol{\varphi}}_{N}$, $\tilde{\chi}_{N}$.

The Hamiltonians $\mathbf{H}_{+}$(4.5) acting in the Heisenberg representation become block-diagonal in the basis of $\tilde{\boldsymbol{\varphi}}_{v}$ and $\tilde{\boldsymbol{\chi}}_{v}$ defined by (4.6). The fermion space splits under its action into the direct sum of two-dimensional invariant subspaces each being a linear span of the pair $\tilde{\boldsymbol{\varphi}}_{v}$ and $\tilde{\boldsymbol{\chi}}_{v}$ with the same $v$. For a diagonalization of the Hamiltonians within each subspace a unitary transformation is required.

Bogoliubov's Transformation. This unitary transformation is identical for both sectors $\mathrm{R}= \pm 1$ :

$$
\begin{aligned}
& \tilde{\boldsymbol{\varphi}}_{v}=\frac{1}{\sqrt{2}}\left(\mathbf{c}_{v} e^{i \alpha_{v}}+\mathbf{c}_{-v} e^{-i \alpha_{v}}\right) \\
& \tilde{\boldsymbol{\chi}}_{v}=\frac{1}{\sqrt{2}}\left(\mathbf{c}_{v} e^{i \beta_{v}}+\mathbf{c}_{-v} e^{-i \beta_{v}}\right),
\end{aligned}
$$


where $c_{v}^{+}, c_{v}$ are creation and annihilation Fermi-operators respectively, both possessing a momentum equal to $p_{v}$. They commute in a standard manner.

$$
\left\{\mathbf{c}_{v}, \mathbf{c}_{\mu}\right\}=\left\{\mathbf{c}_{v}^{+}, \mathbf{c}_{\mu}^{+}\right\}=0 ; \quad\left\{\mathbf{c}_{v}, \mathbf{c}_{\mu}^{+}\right\}=\delta_{\mu, v} .
$$

The form (4.10) of the fermion transformation warrants the relations (4.9) to hold. However, anticommutation relations (4.8) between $\tilde{\boldsymbol{\varphi}}_{v}$ and $\tilde{\chi}_{v}$ impose an additional constraint on the phases in (4.10):

$$
\exp \left[i\left(\alpha_{v}-\beta_{v}+\alpha_{-v}-\beta_{-v}\right)\right]=-1 .
$$

A final determination of phases in (4.10) becomes possible after the Hamiltonians in both sectors $R= \pm 1$ are expressed in terms of $\mathbf{c}_{v}$ and $\mathbf{c}_{v}^{+}$by virtue of (4.10). Requiring then the terms proportional to $\mathbf{c}_{v} \mathbf{c}_{-v}$ and $\mathbf{c}_{v}^{+} \mathbf{c}_{-v}^{+}$to vanish and accounting for (4.11), one comes to the following relations:

$$
\exp \left[2 i\left(\alpha_{v}-\beta_{v}\right)\right]=\exp \left[2 i\left(\alpha_{-v}-\beta_{-v}\right)\right]=\frac{k-\mathrm{e}^{-i p_{v}}}{\mathrm{e}^{i p_{v}}-k} .
$$

The above transformation changes the form of the Hamiltonians $\mathbf{H}_{ \pm}(4.5)$ into the following one:

$$
\begin{aligned}
\mathbf{H}_{ \pm} & =E_{0}^{ \pm} \mathbf{I}+\sum_{v} \varepsilon\left(p_{v}\right) \mathbf{c}_{v}^{+} \mathbf{c}_{v} ; \\
E_{0}^{ \pm} & =-\frac{1}{2} \sum_{v} \varepsilon\left(p_{v}\right),
\end{aligned}
$$

where $E_{0}^{ \pm}$is the ground state energy. By $\varepsilon(p)$ an elementary excitation energy is denoted. It is positive for $p \neq 0$,

$$
\varepsilon(p)=\left(1+k^{2}-2 k \cos p\right)^{1 / 2} \quad \text { if } \quad p \neq 0 ; \quad \varepsilon(0)=1-k .
$$

The critical point corresponds to $k=1$ that yields the excitation spectrum,

$$
\varepsilon(p)=2|\sin (p / 2)| .
$$

Substituting (4.16) into (4.14) one can perform the summation in the expression for the ground state energy and obtains

$$
E_{0}^{+}=-\operatorname{cosec}(\pi / 4 N) ; \quad E_{0}^{-}=-\cot (\pi / 4 N) .
$$

The Conformally Invariant Limit. The relation of the lattice Hamiltonians (4.14) to the conformally invariant ones obtainable from (3.14) can be traced in the following way. Assume $N \gg 1$ and consider the truncated Hamiltonians (4.14) where a cutoff $|v| \leqq v_{0}\left(1 \ll v_{0} \ll N\right)$ is introduced. Expand in the powers of $1 / N$ the ground state energy and the excitation energies in the truncated Hamiltonians leaving the terms of the order $1 / N$; a smallness of the momentums involved $(P \ll 1)$ should be accounted for. The truncated Hamiltonians look as follows:

$$
\mathbf{H}_{ \pm}=-\frac{8 N}{\pi} \mathbf{I}+\left\{\begin{array}{r}
-(\pi / 24 N) \\
(\pi / 12 N)
\end{array}\right\} \mathbf{I}+\frac{\pi}{N} \sum_{v=-v_{0}}^{v_{0}}|v| \mathbf{c}_{v}^{+} \mathbf{c}_{v},
$$

where summation is performed over the half-odd-integer $v$ in $\mathrm{R}=1$ sector and the integer $v$ in $\mathrm{R}=-1$ sector. 
Owing to the Heisenberg equations of motion governed by the Hamiltonians (4.18) the right fermions (i.e. with $v>0$ ) are analytic: $\mathbf{c}_{v} \sim \exp \left[-\frac{\pi}{N} v(t+i n)\right]$. Similarly the left fermions $\mathbf{c}_{v}(v<0)$ are antianalytic. A natural identification is the following one:

$$
\mathbf{c}_{v}=\boldsymbol{\psi}_{v} ; \quad v>0 ; \quad \mathbf{c}_{v}=\overline{\boldsymbol{\psi}}_{v}, v<0 ; \quad R=2 N
$$

Truncating Virasoro generators $\mathbf{L}_{0}$ and $\overline{\mathbf{L}}_{0}$ in (3.14) by a cut-off $v<v_{0}$ one can assure the equivalence of $\mathbf{H}_{+}$with the NS Hamiltonian and that of $\mathbf{H}_{-}$with the Ramond Hamiltonian. A term $-8 N / \pi \mathbf{I}$ is analogous to an infrared-divergent and ultraviolet regularized term in a field theory.

Despite the above algebraic equivalence of the Hamiltonians, their sets of the eigenvectors don't fit into one-to-one correspondence.

This disagreement is caused by topological reasons. Really, Majorana fermions (4.3) acting on a spin state produce a domain wall. But as far as cyclic boundary conditions are imposed on the spin chain only an even number of domain walls may be produced. Thus an even number of fermions may be excited over the ground state.

These heuristic arguments can be put on the more rigorous algebraic ground. Let us remark that a single fermion anticommutes with the spin reversal operator $\mathbf{R}$ (4.2). Clearly an odd number of fermions acting on some state with a definite eigenvalue of $\mathbf{R}$ remove this state out of its sector. From the other viewpoint, a number of states with even numbers of fermions excited in each sector is equal to

$$
\sum_{n=0}^{2 N} C_{2 N}^{2 n}=2^{2 N-1} .
$$

Comparing it with a number of spin states equal to $2^{2 N}$ one can make sure that the states with an even number of fermions exhaust the whole set of eigenstates.

Consider a sector with $\mathrm{R}=1$. Here a ground state is a counterpart of the vacuum state $|0\rangle$ in the conformally invariant theory. The states with an even number of both right and left excited fermions can be identified with conformal descendants of the vacuum vector. The states with an odd number of both right and left excited fermions constitute another conformal modulus. Its ancestor is a vector

$$
|\varepsilon\rangle=\mathbf{c}_{-1 / 2}^{+} \mathbf{c}_{1 / 2}^{+}|0\rangle
$$

related to an operator $\boldsymbol{\varepsilon}$ of the energy density $[3,4]$.

In the sector where $\mathrm{R}=-1$ the ground state may be constructed in two ways:

$$
\mathbf{c}_{0}|\sigma\rangle=0 ; \quad \mathbf{c}_{0}^{+}|\sigma\rangle=|\mu\rangle
$$

or

$$
\mathbf{c}_{0}^{+}|\mu\rangle=0 ; \quad \mathbf{c}_{0}|\mu\rangle=|\sigma\rangle .
$$

Both states $|\sigma\rangle$ and $|\mu\rangle$ are annihilated by $\mathbf{c}_{v}$ with $v \neq 0$.

Below the phase transition point at $k<1$ the state $|\sigma\rangle$ possesses a minimal energy in conformity with (4.13) and (4.14). Above the critical point at $k>1$ the 
state $|\mu\rangle$ becomes a ground one. A permutation of the ground states at $k=1$ is caused by a reversal of sign of the quantity $\varepsilon(0)$ regarded as a function of $k$. In the very critical point at $k=1$ both states are much the same. The prehistory can only select either as a ground state.

In the above discussion (see (4.19) we omitted the problem concerning correspondence between Ramond operators $\psi_{0}$ and $\bar{\psi}_{0}$ and the Ising fermions $\mathbf{c}_{0}$ and $\mathbf{c}_{0}^{+}$in the $\mathrm{R}=-1$ sector. We were able to do that for all these operators fall out of the Hamiltonians. Thus all of them are independent on time and coordinate which prevent from maintaining an unambigous correspondence. One can assume e.g. $\tilde{\boldsymbol{\varphi}}_{0}=\boldsymbol{\psi}_{0}$ and $\tilde{\boldsymbol{\chi}}_{0}=\overline{\boldsymbol{\psi}}_{0}$. A freedom in choosing the phase in the formula (4.10) is analogous to the same freedom in the conformally invariant theory (see (3.9)). We are obliged to note that phase factors for $\psi_{0}$ and $\bar{\psi}_{0}$ according to (4.12) and (4.13) obey the constraint $\exp \left[2 i\left(\alpha_{0}-\beta_{0}\right)\right]=-1$ imposed by anticommutation of $\boldsymbol{\psi}_{0}$ and $\overline{\boldsymbol{\psi}}_{0}$.

Once a ground state is settled, e.g. $|\sigma\rangle$, the excited states are created by an action of an even number of $\mathbf{c}_{v}$ operators. The total multitude of these states is identical to the conformal modulus $|\sigma\rangle$ in the Ramond sector. This set arises when a system arrives into its critical point from below $(k<1)$.

Another conformal modulus is produced when the system approaches its critical point from above $(k>1)$. The excited states are created by an even number of the operators $\mathbf{c}_{v}^{+}$with $v \neq 0$ or by an odd number of $\mathbf{c}_{v}^{+}$with $v \neq 0$ and by $\mathbf{c}_{0}$ acting on $|\mu\rangle$.

\section{The Transfer-Matrix Eigenvalues (TME)}

The Hamiltonian (4.1) commutes with a parametric TM family of the Ising model $[9,10]$. The general TM elements are given by the formula

$$
\left\langle\sigma\left|\mathbf{V}\left(K, K^{\prime}\right)\right| \sigma^{\prime}\right\rangle=\exp \left[\sum_{j=1}^{2 N}\left(K \sigma_{j+1} \sigma_{j}^{\prime}+K^{\prime} \sigma_{j} \sigma_{j}^{\prime}\right)\right] .
$$

The TM $\mathbf{V}\left(K, K^{\prime}\right)$ commutes with the Hamiltonian (4.1) provided that the interaction constants and a parameter $k$ are related as follows $[9,10]$ :

$$
\sinh (2 K) \sinh \left(2 K^{\prime}\right)=k^{-1} .
$$

In the phase transition point where $k=1$ the interaction strengths possess a convenient parametrization

$$
\sinh (2 K)=\tan (\theta / 2) ; \quad \sinh \left(2 K^{\prime}\right)=\cot (\theta / 2) .
$$

This parametrization can be extended out of the critical point in terms of elliptic functions (see $[9,10]$ ). But it won't be necessary further. The only thing we should like to take notice of is that the Hamiltonian (4.1) is twice larger than a logarithmic derivative of $\mathbf{V}(\theta)$ (see (2.1)). This is one reason, and the other will be presented below to modify a definition of the transfer-matrix as follows

$$
\mathbb{W}\left(K, K^{\prime}\right)=\mathbf{V}^{T}\left(K^{\prime}, K\right) \mathbb{V}\left(K, K^{\prime}\right)
$$


where a superscript $T$ indicates on a transposition of the TM. A transposed TM $\mathbf{V}^{T}\left(K^{\prime}, K\right)$ may be expressed through the TM $\mathbf{V}\left(K, K^{\prime}\right)$ using a translation operator $[9,10]$,

$$
\mathbf{V}^{T}\left(K^{\prime}, K\right)=\exp (-i \mathbf{P}) \mathbf{V}\left(K, K^{\prime}\right) .
$$

Here $\mathbf{P}$ denotes the operator of momentum as previously. Due to (5.5) TM $\mathbf{W}\left(K, K^{\prime}\right)$ unite into a parametric commutative family provided the interaction constants are related by (5.2). The Hamiltonian (4.1) can be represented in agreement with (2.1) through $\mathbf{W}(\theta)$. The whole TM family $\mathbf{W}(\theta)$ turns into its diagonal form in the basis formed by the eigenvectors of the Hamiltonian (4.1). Just as for the Hamiltonian (4.1), the Jordan-Wigner fermions (4.3) can be viewed as a basis for the Heisenberg representation of the TM W $(\theta)$. Now they obey discrete (in time) equations of motion governed by the $\mathrm{TM} \mathbf{W}(\theta)$. These lattice equations are linear and even diagonal in the basis of fermions $\mathbf{c}_{v}(4.10)$. In other words,

$$
\mathbf{c}_{v} \sim \exp \left(-t \Omega_{v}-i x p_{v}\right)
$$

where

$$
\Omega_{v}=2 \ln \left[\frac{1+\sin \theta\left|\sin \left(p_{v} / 2\right)\right|}{\cos \left(p_{v} / 2\right)-i \cos \theta \sin \left(p_{v} / 2\right)}\right] .
$$

There is no need to present here all the cumbersome calculations leading to (5.6). The final result can be extracted out of the book by Baxter [9] (see (7.9.7)). According to the cited formula,

$$
\ln \left(\Lambda_{n} / \Lambda_{0}\right)=-\sum_{j} \Omega_{v_{1}},
$$

where the quantum numbers $v_{j}$ take their values in conformity with the prescription (4.7). Recall that the number of terms in the sum (5.7) is always even. The TME (5.7) belongs to the eigenvector of the Hamiltonian (4.1) specified by the quantum numbers $v_{i}$. The time evolution of the state $|n\rangle$ is governed by a power law $\left(\Lambda_{n} / \Lambda_{0}\right)^{t}$ justifying (5.6).

A logarithm of the maximal TM in agreement with reference [9] (see (7.9.15) and (7.10.1)) is equal to the following sum

$$
\ln \Lambda_{0}^{ \pm}=\sum_{v} \Phi\left(p_{v} ; \theta\right),
$$

where the superscripts " \pm " denote $R= \pm 1$ as previously and the array of summation is chosen in accordance with (4.7). The function $\Phi(p ; \theta)$ in (5.8) has the following appearance:

$$
\Phi(p ; \theta)=\ln \{4[\operatorname{cosec} \theta+|\sin (p / 2)|]\} .
$$

As we know now an expansion in powers of $1 / N$ leads to the conformally invariant limit of the theory. This expansion can be performed immediately for the quantities $\Omega_{v}$ :

$$
\Omega_{v}=\sin \theta \cdot\left|p_{v}\right|+i \cos \theta \cdot p_{v} .
$$


However, more subtle methods are needed for the logarithms of the maximal TM eigenvalues. That may be an asymptotic formula by Euler-Maclaurin or a method of resummation by Poisson (see [9]) with a subsequent asymptotic estimation of the high Fourier harmonics of $\Phi(p, \theta)$ in variable $p$. The result reads

$$
\ln \Lambda_{0}^{ \pm}=2 N f(\theta)+\left\{\begin{array}{r}
(\pi / 24 N) \sin \theta \\
-(\pi / 12 N) \sin \theta
\end{array}\right\} .
$$

The superscripts " \pm " mean as usual $\mathrm{R}= \pm 1$ and the higher number in the braces should be attributed to the $\mathrm{R}=1$ sector while the lower one - to the $\mathrm{R}=-1$ sector. A function $f(\theta)$ in (5.11) is equal to the thermodynamic value of a free energy density

$$
f(\theta)=\int_{0}^{\pi} \frac{d p}{\pi} \Phi(p, \theta) .
$$

The expansions (5.10) and (5.11) are in perfect agreement with the general conjectures (1.2) and (2.21) provided that the parameters in the former fit the following values:

$$
v=1 ; \quad \alpha=\pi / 2-\theta \quad \text { and } \quad R=2 N \text {. }
$$

\section{A Conformally Invariant Counterpart of the Ising Model on a Parallelogram}

In the present section we study a conformally invariant theory of the free Majorana fermions occupying a parallelogram and obeying periodic or antiperiodic boundary conditions. It will be shown that a free energy $\mathscr{F}$ of such a system considered as a function of the vectors $\mathbf{R}_{1}$ and $\mathbf{R}_{2}$ forming the parallelogram depends only on the ratio of their lengths $R_{2} / R_{1}$ and an angle $\varphi$ between $\mathbf{R}_{1}$ and $\mathbf{R}_{2}$. It will be of great convenience to realize the above two quantities as a modulus and a phase of some complex number $\tau$ :

$$
\tau=r e^{i \varphi} ; \quad \bar{\tau}=r e^{-i \varphi} ; \quad r=\mathbf{R}_{2} / \mathbf{R}_{1} .
$$

As it will be demonstrated a little bit later, the dependence of the free energy on $\tau$ and its conjugate $\bar{\tau}$ splits into analytic and antianalytic parts

$$
\mathscr{F}(\tau, \bar{\tau})=F(\tau)+\bar{F}(\bar{\tau}) .
$$

Let us examine a response of the free energy $\mathscr{F}\left(\mathbf{R}_{1}, \mathbf{R}_{2}\right)$ when $\mathbf{R}_{1}$ and $\mathbf{R}_{2}$ undergo some transformation. First we observe that any transformation of $\mathbf{R}_{1}$ and $\mathbf{R}_{2}$ can be reduced to some linear coordinate transformation. Remembering the conformal invariance one should exclude the conformal constituent out of the transformation studied. In other words, an identical dilatation or rotation of both vectors $\mathbf{R}_{1}$ and $\mathbf{R}_{2}$ cannot change the value of the free energy. For this reason the latter depends only on $r$ (see (6.1)) and $\varphi$ or $\tau$ and $\bar{\tau}$.

Now let us concentrate on the variation of the free energy induced by an infinitesimal coordinate transformation leaving one of the vectors, e.g. $\mathbf{R}_{1}$ invariant. Exploiting once more a conformal invariance we choose $\mathbf{R}_{1}$ as a unit vector along the axis of abscissa. The second vector $\mathbf{R}_{2}$ will be specified using polar coordinates $r$ and $\varphi$. In the Cartesian frame $(\tau, \sigma)$ both vectors and a general 
variation of $\mathbf{R}_{2}$ look as follows:

$$
\begin{gathered}
\mathbf{R}_{1}=\left(\begin{array}{l}
1 \\
0
\end{array}\right) ; \quad \mathbf{R}_{2}=r\left(\begin{array}{c}
\cos \varphi \\
\sin \varphi
\end{array}\right) ; \\
\delta \mathbf{R}_{2}=\delta r\left(\begin{array}{l}
\cos \varphi \\
\sin \varphi
\end{array}\right)+r \delta \varphi\left(\begin{array}{r}
-\sin \varphi \\
\cos \varphi
\end{array}\right) .
\end{gathered}
$$

A general linear infinitesimal coordinate transformation $\mathscr{A}$ satisfying the above conditions, i.e.

$$
\mathscr{A} \mathbf{R}_{1}=0 ; \quad \mathscr{A} \mathbf{R}_{2}=\delta \mathbf{R}_{2},
$$

may be represented in the following form:

$$
\mathscr{A}=\frac{\delta r}{r}\left(\begin{array}{cc}
0 & \cot \varphi \\
0 & 1
\end{array}\right)+\delta \varphi\left(\begin{array}{cc}
0 & -1 \\
0 & \cot \varphi
\end{array}\right) .
$$

A response of the free energy for this transformation should be expressed as usual through the average ST value:

$$
\delta \mathscr{F}=\int \frac{d^{2} x}{2 \pi}\left\langle 0\left|T_{\mu \nu}(\mathbf{x})\right| 0\right\rangle \mathscr{A}_{\mu \nu},
$$

where the integration is performed over the parallelogram formed by the vectors $\mathbf{R}_{1}$ and $\mathbf{R}_{2}$ (6.3). Writing $\delta \mathscr{F}$ in the form (6.6) we accounted for the linearity of the coordinate transformation and the symmetry of ST. As will be shown below, the average ST value is coordinate independent. The integration in (6.6) reduces therefore to the multiplication of the integrand by the value of the parallelogram area equal to $r \sin \varphi$. The last remark results in the following expression for $\delta \mathscr{F}$ :

$$
\begin{aligned}
\delta \mathscr{F}= & (\delta r / 2 \pi)\left(\left\langle T_{\tau \sigma}\right\rangle \cos \varphi+\left\langle T_{\sigma \sigma}\right\rangle \sin \varphi\right) \\
& +(r \delta \varphi / 2 \pi)\left(-\left\langle T_{\tau \sigma}\right\rangle \sin \varphi+\left\langle T_{\sigma \sigma}\right\rangle \cos \varphi\right) .
\end{aligned}
$$

Substituting the Cartesian frame ST elements through its analytic and antianalytic components by virtue of (2.6) and (2.7) we arrive finally at the formula

$$
\delta \mathscr{F}=(i / 2 \pi)(\langle T\rangle \delta \tau-\langle\bar{T}\rangle \delta \bar{\tau})
$$

where $\tau$ and $\bar{\tau}$ are the same as in (6.1).

Now we proceed to the computation of the ST average in a specific model of Majorana fermions examined previously (Sect. 3) for a strip geometry. Our calculation will be based on the operator product expansion (3.10). Hence an explicit expression for a pair correlation of periodic or antiperiodic fermions is required.

A Green function $\langle\psi(z) \psi(\zeta)\rangle$ is meromorphic in $z$, double periodic and possessing a single simple pole in the parallelogram formed by (half-) periods equal to 1 and $\tau$. According to the Liouville theorem, both numbers 1 and $\tau$ cannot be the periods of the Green function. At least one of them must be an antiperiod. Therefore three types of boundary conditions are possible. We shall denote by subscript $+(-)$ antiperiodic (periodic) boundary conditions imposed along the period 1. Similarly, by $+(-)$ superscript the antiperiodicity (periodicity) along the second period $\tau$ will be denoted. 
Once some definite type of periodicity is chosen, the correlation function $\langle\psi(z) \psi(\zeta)\rangle$ is determined accounting for the value of residue in the pole equal to unity. Though up to now we discussed only $z$ dependence of the pair correlation, no special study of the second argument dependence is required. Really, being translationally invariant $\langle\psi(z) \psi(\zeta)\rangle$ can depend on a single argument $(z-\zeta)$ only.

The explicit expressions for the fermion Green functions will be represented in terms of the Jacobi elliptic function of modulus $k$ related with $\tau$ in a usual manner [18] (Sect. 13.20)

$$
k=\frac{\vartheta_{2}^{2}(0, \tau)}{\vartheta_{3}^{2}(0, \tau)} ; \quad k^{\prime}=\frac{\vartheta_{4}^{2}(0, \tau)}{\vartheta_{3}^{2}(0, \tau)} ; \quad k^{2}+k^{\prime 2}=1 .
$$

Here $\vartheta_{2}, \vartheta_{3}, \vartheta_{4}$ denote the standard Jacobi, theta-functions [18] (Sect. 13.20) and $k^{\prime}$ is the modulus complementary to $k$. Finally, we exhibit the Green functions involved,

$$
\begin{aligned}
& \langle\psi(z) \psi(\zeta)\rangle_{+}^{+}=2 K(k) \mathrm{ds}(2 K(k)(z-\zeta), k) ; \\
& \langle\psi(z) \psi(\zeta)\rangle_{+}^{+}=2 K(k) \mathrm{ns}(2 K(k)(z-\zeta), k) ; \\
& \langle\psi(z) \psi(\zeta)\rangle_{+}^{+}=2 K(k) \operatorname{cs}(2 K(k)(z-\zeta), k) .
\end{aligned}
$$

Here $K^{\prime}(k)$ designates a full elliptic integral of the first kind and of modulus $k^{\prime}$ [18] (Sect. 13.8). As for the nomenclature of the elliptic functions, one can consult ref. [18] (Sect. 13.9).

The Laurent series for every function (6.10)-(6.12) includes only odd powers of $(z-\zeta)$ in agreement with an operator product expansion (3.10). Extracting the linear in $(z-\zeta)$ term of these series one obtains an average ST value $\langle T\rangle$ for any type of boundary conditions (see [18] Sect. 13.17),

$$
\begin{gathered}
\langle T\rangle_{+}^{+}=\frac{1-2 k^{2}}{12}(2 K(k))^{2} ; \\
\langle T\rangle_{+}^{-}=\frac{1+k^{2}}{12}(2 K(k))^{2} ; \quad\langle T\rangle_{+}^{+}=\frac{k^{2}-2}{12}(2 K(k))^{2} .
\end{gathered}
$$

To remove the variation in (6.8) we express $\delta \tau$ through $\delta\left(k^{2}\right)$ by means of the formula (see [18] Sect. 13.9)

$$
\frac{d \tau}{d k^{2}}=-i \pi\left[2 K(k) k k^{\prime}\right]^{-2} ; \quad \tau=i K^{\prime}(k) / K(k) .
$$

Here $K^{\prime}(k)$ is a full elliptic integral of the first kind but of the modulus $k^{\prime}$ complementary to $k$. The Legendre identity [18] (Sect. 13.9) was used to differentiate with respect to $k^{2}$.

Inserting $\delta \tau=\left(d \tau / d k^{2}\right) \delta\left(k^{2}\right)$ into (6.8) and substituting the ST values (6.13) in it we derive the following expressions for the $\tau$-dependent part of the free energy.

$$
\begin{aligned}
& \mathscr{F}_{+}^{+}=\frac{1}{12} \ln \left|k^{\prime}\right|^{2}=\frac{1}{6} \ln \left|\vartheta_{2}(\tau) \vartheta_{4}(\tau) \vartheta_{3}^{-2}(\tau)\right|^{2} ; \\
& \mathscr{F}_{+}^{-}=\left.\frac{1}{12} \ln |k| k^{\prime 2}\right|^{2}=\frac{1}{6} \ln \left|\vartheta_{2}(\tau) \vartheta_{3}(\tau) \vartheta_{4}^{-2}(\tau)\right|^{2} ; \\
& \mathscr{F}_{-}^{+}=\left.\frac{1}{12} \ln \left|k^{\prime}\right| k^{2}\right|^{2}=\frac{1}{6} \ln \left|\vartheta_{3}(\tau) \vartheta_{4}(\tau) \vartheta_{2}^{-2}(\tau)\right|^{2} .
\end{aligned}
$$


Here we omitted the $u$-dependence for $\vartheta_{i}(u, \tau)$ since $u \equiv 0$. The relations $(6.9)$ have been used.

A remarkable propriety of the expressions (6.15)-(6.17) concerns their permutations induced by modular transformations of $\tau$ [18] (Sect. 13.22). When $\tau$ is replaced by $\tau+1$, an interchange of $\mathscr{F}_{+}^{+}$and $\widetilde{F}_{+}^{-}$takes place while $\mathscr{\mathscr { F }}_{-}^{+}$remains invariant. A transformation $\tau \rightarrow-1 / \tau$ interchanges $\mathscr{F}_{-}^{+}$with $\mathscr{F}_{+}^{-}$leaving $\mathscr{F}_{+}^{+}$ intact. It is important that the total statistical sum of the model is invariant under modular transformations of $\tau$. Really, averaging over all possible boundary conditions is identical to summing over all $\exp \left(-\widetilde{\mathscr{F}}_{\beta}^{\alpha}\right)$ where $\alpha$ and $\beta$ take their values \pm independently.

Let us analyse the limit $\tau \rightarrow 0$ for the formulas (6.15)-(6.17). In this limit one should be able to reproduce the results for the free energy in a strip geometry elaborated in Sect. 3. However, to convert a parallelogram formed by complex numbers 1 and $\tau$ into a strip of a width equal to $R$ along the imaginary axis and inclined at angle $\alpha=\frac{\pi}{2}-\arg \tau$ to the real axis (Fig. 1) one should precede passing to the limit $\tau \rightarrow 0$ by a rescaling of a complex plane $z$ and the corresponding rescaling of $\psi(z)($ see [3])

$$
z=-i \frac{\tau}{R} u ; \quad \psi(z)=\sqrt{\frac{-i \tau}{R}} \tilde{\psi}(u) .
$$

The periods 1 and $\tau$ under the rescaling transmute into $i R / \tau$ and $i R$ respectively, and one can safely let $\tau$ tend to zero. In this limit $\langle\tilde{\psi}(u) \tilde{\psi}(v)\rangle_{+}^{+}$coincide with $\langle\tilde{\psi}(u) \tilde{\psi}(v)\rangle_{-}^{+}$and tend to a higher singular term in the braces while $\langle\tilde{\psi}(u) \tilde{\psi}(v)\rangle_{-}^{+}$ tends to the lower one of the expansion (3.11). Note that the limit $\tau \rightarrow 0$ is equivalent to $k \rightarrow 1$ [18] (Sect. 13.20).

The rescaling cannot cause variation in a free energy as was mentioned above. But at $\tau \rightarrow 0$ the free energy diverges. To handle this singularity, it is convenient to make at first the modular Jacobi transformation $\tau \rightarrow-1 / \tau[18]$ (Sect. 13.22). Then using infinite product representation for theta-functions [18] (Sect. 13.19 formula (16)) one can easily obtain the leading terms of expansion in terms of small $\tau$ for the free energy,

$$
{\mathscr{F}_{+}^{+}}^{+}=\mathscr{F}_{+}^{-}=-\frac{1}{2} \mathscr{F}_{-}^{+}=\frac{\pi}{12} \operatorname{Im}\left(\frac{1}{\tau}\right)+\frac{1}{3} \ln 2 .
$$

The accuracy of this expansion is exponential, i.e. $\exp \left(-\frac{i \pi}{\tau}\right)$. According to (6.1) the right-hand side of (6.19) is proportional to $R_{1}$. Hence the value of the free energy per unit length along the direction of $\mathbf{R}_{1}$ is given by the following expression:

$$
f_{+}^{+}=f_{+}=-\frac{1}{2} f_{-}^{+}=-\frac{\pi}{12 R_{2}} \sin \varphi
$$

This result agrees with the formulas (1.2) and (5.11) once $\varphi=\theta=\pi / 2-\alpha ; v=1$.

\section{Discussion}

In the previous sections we have carefully analysed an expansion in powers of $1 / N$ for the Hamiltonian and transfer-matrices of the Ising chain consisting of $N$ sites 
$(N \gg 1)$. The terms in this expansion proportional to $1 / N$ have been demonstrated to be closely related to the conformally invariant theory. Namely, the corresponding term in the Hamiltonian can be transformed into the regularized conformally invariant free fermion Hamiltonian. The eigenvalues of the momentum and of the Hamiltonian (or of the TM) have been expressed through the central charge and the conformal dimensions in the field theory.

Let us speculate which details of this analysis may be extended to the whole class of the exactly solvable models and which are specific for the Ising model. It seems that the Ising model is unique as far as an explicit operator construction of the effective Hamiltonian for the low lying excitations is concerned. Really, even for the Ising model this construction is associated with a non-local transformation of the initial spin variables. Besides, one should anticipate that a non-trivial renormalization will arise for the other models having conformally invariant counterparts with an intrinsic interaction.

However, we believe that a general scheme presented in Sect. 2 and concerning eigenvalues of the Hamiltonian momentum and TM will persist for the other exactly solvable models. The main point in this scheme is the relation of the conformal dimensions to the momentum of the elementary excitations. Our next aim is to develop the formalism applicable for the evaluation of these quantities in the framework of Bethe ansatz.

An important formula (1.2) is certainly a general one, because no assumptions specific for the Ising model have been used to derive it. A direct geometrical interpretation of the TM spectral parameter as a value of an angle between two periods is indicated in Sect. 6 . This allows to presume that the relation $\theta=\pi / 2-\alpha$ is valid in general. Nevertheless, further examination is required.

Acknowledgements. We are grateful to M. A. Bershadsky, A. M. Polyakov, A. M. Tsvelik and especially to V. A. Fateev and V. L. Pokrovsky for the enlightening discussions.

Note added in proof. After submission of the paper we became aware that the results of Cardy et al. [13] were simultaneously obtained by Affleck [19]. We were also informed that Bogoliubov et al. [20] evaluated some dimensions for the XXZ-model in a transverse magnetic field using finite-size corrections to the energies of the lowest excitations. We are grateful to N. Yu. Reshetikhin for communicating the results prior to publication.

\section{References}

1. Polyakov, A.M.: JETP Lett. 12, 538 (1970)

2. Polyakov, A.M.: JETP 66, 23 (1974)

3. Belavin, A.A., Polyakov, A.M., Zamolodchikov, A.B.: Nucl. Phys. B 241, 333 (1984); J. Stat. Phys. 34, 763 (1984)

4. Dotsenko, Vl.S.: Nucl. Phys. B 235, 54 (1984); J. Stat. Phys. 34, 781 (1984)

5. Dotsenko, Vl.S., Fateev, V.A.: Nucl. Phys. B 240 [FS 12], 312 (1984); Ibid 251 [FS 13], 691 (1985)

6. Knizhnick, V.G., Zamolodchikov, A.B.: Nucl. Phys. B 247, 83 (1984); Zamolodchikov, A.B., Fateev, V.A.: Yad. Fiz. 43, (4) 1031 (1986)

7. Zamolodchikov, A.B., Fateev, V.A.: JETP 89, 380 (1985)

8. Andrews, G.E., Baxter, R.J., Forrester, P.J.: J. Stat. Phys. 35, 193 (1984)

9. Baxter, R.J.: Exactly solved modes in statistical mechanics. London: Academic Press 1982

10. Pokrovsky, S.V., Bashilov, Yu.A.: Commun. Math. Phys. 84, 103 (1982) 
11. Fateev, V.A., Zamolodchikov, A.B.: Phys. Lett. A 92, 37 (1982)

12. Cardy, J.L.: In: Phase transitions and critical phenomena, Vol. 11. Domb, C., Lebowitz, J. (eds.). New York: Academic Press 1986

13. Blötte, H.W.J., Cardy, J.L., Nightingale, M.P.: Conformal invariance, the central charge and universal finite-size amplitudes in two-dimensional theories. Phys. Rev. Lett. 56, 716 (1986)

14. Nightingale, M.P., Blötte, H.W.J.: J. Phys. A 16, L657 (1983); Privman, V., Fisher, M.E.: Phys. Rev. B 30, 322 (1984)

15. Neveu, A., Schwartz, J.M.: Nucl. Phys. B 31, 86 (1971)

Ramond, P.: Phys. Rev. D 3, 2493 (1971)

16. Onsager, L.: Phys. Rev. 65, 117 (1944)

Kaufman, B.: Phys. Rev. 76, 1232 (1949)

17. Bashilov, Yu.A., Pokrovsky, S.V.: Commun. Math. Phys. 76, 129 (1980)

18. Bateman, H., Erdelyi, A.: Higher transcedental functions, Vol. 3. New York, Toronto, London: McGraw-Hill 1955

19. Affleck, I.: Phys. Rev. Lett. 56, 746 (1986)

20. Bogoliubov, N.M., Isergin, A.G., Reshetikhin, N.Yu.: JETP Lett. 44, (9) 405 (1987)

Communicated by Ya. G. Sinai

Received September 10, 1986 